\title{
DESCRIBING THE TACTICAL KNOWLEDGE USED BY YOUNG COMPETITIVE SOCCER PLAYERS: A PSYCHOPHENOMENOLOGICAL ANALYSIS
}

original paper

() Wroclaw University of Health and Sport Sciences

DOI: https://doi.org/10.5114/hm.2022.108323

\author{
GRÉGORY HALLÉ PETIOT ${ }^{1 \oplus}$, RAFAEL BAGATIN ${ }^{2 \oplus}$, ALAIN MOUCHET ${ }^{3 \oplus}$ \\ ${ }^{1}$ Laval University, Quebec, Canada \\ ${ }^{2}$ Faculty of Sports, University of Porto, Porto, Portugal \\ ${ }^{3}$ Department of Sporting and Physical Activity Techniques and Sciences, Paris-East Créteil University, Créteil, France
}

\begin{abstract}
Purpose. Decision-making is the process through which players choose the most appropriate action to perform in the play. Previous investigations did not clearly portray the specific decisional background of learning players considering the progressing state of their capabilities and game knowledge. The study aimed to describe significant information picked up in situ and how young soccer players applied it to make decisions in the play.
\end{abstract}

Methods. Three male soccer players aged 14 years were interviewed after 2 official district championship games in Portugal. Their games were filmed; the video sequences showing offensive actions were extracted and edited for visualization. Before questioning, each sequence was visualized for recalling the game actions. The explicitation interview technique was used to help the athletes describe in detail their recalled actions. In line with the recommendation in similar studies, a content analysis of the interviews was conducted to identify the decisional background and the links between elements of information picked up in situ and the decision itself.

Results. The players did not perform a detailed judgement for every decision and were influenced by direct constraints such as opponent pressure. In contrast, they occasionally assessed risks and opportunities emerging in the game depending on their colleagues' actions and the pitch zone. At times, they relied on their imagination of what their teammates would do with the action outcome.

Conclusions. Key elements of the decisional background are common among learning players and can be used as a reference for further investigation or practical intervention in game teaching.

Key words: decision-making, tactics, team sports

\section{Introduction}

Performance in team sports is deeply analysed to seize what makes teams win and, more precisely, how players organize to overcome the opposition [1]. This implies that all players need to 'know what to do' to overcome the opposition and manage cooperation with teammates, whilst making collective organization work. An important field of research in sports sciences supports the idea that all of the doing stems from the decisional process [2].

According to traditional models, decision-making comprises perception, information processing, and the generation of a solution to execute, which, in the context of play, all happen in a very limited time [3].
To do so, experienced players reportedly use anticipation to gain a time in advance in the play, to direct their attention towards key stimuli, in addition to evaluating adequately what they perceive [4-6]. Learning players, for their part, make decisions by prioritizing what they can do in the time they afford and with the abilities they master, which reflects a first major challenge for young players [7]. Whilst clearer models of the decisional process have helped understand how experts decide, they also hint the actual lived experience of young, learning players.

Even if player development is strongly directed by the teaching of several aspects of performance that progressively give a competitive advantage, this development is still characterized by its nonlinear nature [8].

Correspondence address: Grégory Hallé Petiot, Laval University, Rue de la Terrasse, Québec, Canada, G1V 0A6, e-mail: ghpetiot@icloud.com, https://orcid.org/0000-0003-3524-7890

Received: September 23, 2020

Accepted for publication: April 27, 2021

Citation: Petiot GH, Bagatin R, Mouchet A. Describing the tactical knowledge used by young competitive soccer players: a psychophenomenological analysis. Hum Mov. 2022;23(4):99-111; doi: https://doi.org/10.5114/hm.2022.108323. 
Players and their experience, the understanding and knowledge of the game can therefore hardly be compared; all the content that players may use to make decisions would initially be personal and subjective [9]. On the other hand, athletes still have to organize altogether to solve the situations that occur in the play $[1,10]$. As a result, those who subscribe to such organization with the aim to earn a competitive advantage should build models that help them synchronize their actions [11]. In sum, the decisional background is subjective and individual but is also shared by the members of a team that is committed to organize.

In that sense, we understand that high-performing youth players competing in championships may adopt common references, although not all will necessarily assimilate or integrate them whilst they are still building their own understanding of the game itself. Considering the nonlinear nature of learning of the game and the variation in the assimilation of the common organizational references, up to this date, no data offer a detailed map of the understanding and knowledge of the game that is used or should be assimilated to make the best decisions in the play. Despite the difficulty to elaborate such a map, there is still room to describe the decisional background of learning soccer players on the basis of their own reality.

Investigations on the decisional background of team sports players were conducted in the past, utilizing several approaches that help grasp the various components of decisions [10, 12]. Amongst the emerging investigation approaches, psychophenomenology begins with a subject's very own point of view. More specifically, this approach consists in emphasizing the subjectivity of decisions as empiric data for investigation on the elements that support decisions. According to the instigators of associated techniques, the lived experience reflects a representative starting point to study decision-making and can be investigated in team sports [13-15].

In addition to starting from the performer's lived experience, the approach has, to our conception, the capability to help discern judgement calls, cognitive mechanisms, and thoughts at the origin of decisions in the play [16]. Amongst the techniques associated with the psychophenomenological approach, the explicitation interview offers the opportunity to explore elements of information that were processed by a player during the making of a decision and that potentially reveal their knowledge and understanding of the play. Finally, we assume that the description of the lived experience of different players in different situations of play with this technique could embed common in- formation that might be used as a reference for both research and practice.

The explicitation interview technique has been used to investigate decisions in soccer $[10,17]$ although it has not specifically led to portray the experience of learning players. In other sport modalities, the technique was used among a few players only [18]. On the other hand, holistic approaches to teaching games, also based on questioning, were reportedly used in scientific experiments with developing players; the results showed improvement in actions in the play $[14,19]$ but did not inform about the decisional background. In sum, investigations either for research or for practice still miss more detailed identification of key elements of information that reflect the lived experience and decisional process, specifically in learning players. Such information would help researchers compare better and educators teach the game with respect to the information that makes sense to the player if having a reference in hands.

Thus, the aim of this study was to describe the elements of information picked up in situ (referred to as 'cues') that were significant to young soccer players and how the athletes would put them in relation to make decisions in the play. These elements of information and how they were processed suggestively reflect conceptions of the game (i.e., knowledge and understanding), as well as the cognitive mechanisms solicited during the decisional process [20, 21]. More specifically, analysing the content of judgement was assumed to lead to the decisional background for key actions in the play.

\section{Material and methods}

\section{Participants}

Three male soccer players aged 14 years representing a first regional division soccer club in the district of Porto in Portugal were interviewed 2 times each about actions they had performed in official championship games less than 3 days before the interviews. Filming and interviewing 2 times guaranteed the researchers to reach a minimum of actions amidst the uncertainty of the selected players to play both games and allowed to compare actions and answers between matches and interviews.

All participants played in the same team, trained soccer with their team at least 2 times a week in formal team training sessions, and would play weekly games in the local district championship following official FIFA rules. All were affiliated to a club with support- 
ing registration documentation and the team was supervised by a level 1 certified coach, both in conformity with the rules of the Portuguese Soccer Federation. In addition to being in the starting line-up, all the 3 participants had been practising football for an average of 7 years, mostly in the same club that they were representing during the study.

In order to analyse as many offensive actions in the play as possible, we selected offensive players who were recommended by the coach within the offensive sector of the team shape. As a result, we filmed and interviewed 2 offensive midfielders and 1 striker. Further details on the sample are provided in the study protocol.

\section{Instruments}

The protocol is in line with the composition of the video and interview process elaborated and utilized in similar research in rugby [15, 22]. The researchers selected attacking actions that were significant for ball recovery or build-ups in continuous sequences of play, discarding set plays and throw-ins. The players were shown videos of their own actions in the play and were guided to describe what happened. The videos were filmed with a GoPro Session camera installed on a pole behind the opponent goal, which provided an angle of view from 20' high and allowed to observe the whole field with the exception of the corners of the offensive midfield. The images were processed on an Apple MacBook Pro with the Apple iMovie software. The interviews were recorded with the MacBook microphone; the audio files were manually transcribed and each narrative was chronologically reconstituted with text editing software. Atlas version 8 for Apple Mac computers was used to tag segments and extract relevant examples.

\section{Protocol and procedures}

During both filmed games, the researcher and his assistant noted the time of every occasion that any of the selected players had the ball in their possession. Only attacking actions that contributed to the play or allowed the team to maintain ball possession were taken on owing to their significance to the players. After the game, the researcher extracted the listed sequences of play from the video file. A video with the corresponding sequences was assembled and edited with a computer movie-editing software. Each action was edited so that the beginning of each sequence of play would begin with a 2-second fixed frame to help the recall of the situation of play [23].
The corresponding video sequences were shown to the participants to stimulate the recall of their involvement in the play [24]. The explicitation interview [25] was used to question the players about their action in each sequence of play, without the support of the video. After the visualization of every sequence, the screen was shut down and the participant would naturally begin to talk about his action with the ball and its context in the game. Afterwards, the researchers would ask questions with the intention to make the athletes explore the sensations and mental actions they could have experienced in the action [25]. To help the players recall the actual action, they would ask such questions as: 'How does the ball get to you?,' 'When you touch the ball, what do you do?,' 'How do you do to [action mentioned as the answer to the previous question]?'. This step was intended to collect more information about the actual action, which the researcher would refer to in his following questions. The aim of the new questions was to explore the tactical content of the actions without referring to the precise moment or asking 'why a decision was made'. In order to obtain a useful degree of details, all questions were based on previous answers. For example, the interviewee would mention to see his teammate and the researcher would ask a general question about the teammate. Questions were asked to lead the interview to reveal more about him without examining specific details. According to the creators of the protocol, all questions based on the lived experience without querying specific information favour a more reliable recall as the interviewee describes what they encountered [26].

In general, the interviews were conducted 2 hours after the player's latest performance to optimize recall and were performed in a calm environment in the presence of the assistant researcher. All participants were interrogated 2 times for a maximum duration of 40 minutes in a lap of 2 weeks. Each interview allowed to cover at least 8 actions that they had performed in the game just before. In addition to directly involving the interrogated players, each action was selected with the use of the same criteria defined in a similar study: the performed actions were 'efficient in provoking misbalance in the opponent team, in keeping ball possession, to give sequence to the play to the point of leading to scoring a point' [27, p. 191]. Plus, the researchers judged if an action was significant to the participants if they reacted, affirmed that they recalled it, and described it with ease as they visualized it. This helped the researchers cover up to 8 significant actions per game per participant, as stated in the objectives. 
The method was previously tested and adapted to the age of the participants by simplifying the questions and vocabulary but still to generate an access to their pre-reflected consciousness and make them re-experiment the situations of play. The questions also directed the interviewees to recall and describe their lived experience in order to avoid embellishment of the narratives or the repetition of pre-formulated action rules as much as possible. The researcher participated in 2 complete formations of 12 hours each and implemented methodological try-outs prior to data collection.

The data analysis was intended to identify the cues that were considered the most important when the participants made their decisions in the play. All the 6 interviews were transcribed, and the transcriptions were verified by external collaborators. Their content was studied by using a qualitative content analysis protocol, combining verbal reports and customized coding strategies to optimize reaching the highest level of details [28, 29]. First, each scene was chronologically reconstituted in a table made of 3 rows (before, during, and after the action), as shown in Table 1 [22]. This allowed to filter the segments, discard repetitions, and classify information in 3 columns: perceptions, information processing, and actual motor actions. Since the promise of phenomenology is to recognize the subjective experience, the coding was solely conducted to emphasize recurrent, common elements of information, without the aim to compare. It was evaluated that the experimental design of the study could identify commonalities since the tactical nature of the experience is always based on the same information: ball, teammates, opponents, space [30]. The perceptions referred to: (a) cues that were named by the interviewees; (b) information processing referred to any judgement, relation created between cues, or thoughts; and (c) actual execution of actions. Actions, on their end, referred to the description of act. Special attention was given to the distinction between motor actions, i.e., body or technical movements, and mental actions, i.e., perceptions, thoughts, or internal dialogue (self-talk). The distinction of these narrative elements was essen-

Table 1. Chronological reconstitution template of the lived experience

\begin{tabular}{llll}
\hline Time & Perceptions & $\begin{array}{c}\text { Information } \\
\text { processing }\end{array}$ & Actions \\
\hline
\end{tabular}

Before

During

After tial to a clear analysis and interpretation of the players' lived experience of each situation of play.

The final reconstitution tables were then processed in a qualitative analysis software for the assignment of codes to relevant segments of the narratives. The coding was implemented with the aim to group cues, information processing mechanisms, and actions, given that all this content comes together. Thus, a code was assigned to each segment in which the interviewee would directly refer to, for instance, the displacement of his teammate. The objective of the detailed, grouped coding was to portray the lived experience behind each decision, including actions and thoughts [31]. Particular attention was turned to identify: (a) the set of cues that the decisions were based on; (b) their decisional background, including, for instance, hints of learnings, preferences, intentions, or even directives; and, finally (c) conceptions of the game [20,32].

The codes were grouped in accordance with their purpose and richness. First, the simple stimuli such as single cues were identified, as, for instance, a teammate. Furthermore, some other concepts that the players referred to were a combination of cues since they were appreciated altogether, simultaneously, as in the example: 'an unmarked teammate running forward'. Finally, information processing, including judgements, and external references such as action rules were extracted from the transcriptions to create relations with the perceptions.

The list of codes was specifically elaborated with respect to both the structure of the game of soccer and the concepts collected during the preliminary methodological try-outs [33]. The list was built by the researcher, reviewed by the assistant researcher, corrected by the researcher and the assistant together, and submitted to the last expertise by a qualitative researcher at the Faculty of Sports of the University of Porto. This process helped mitigate ambiguities and agree on the terms, definitions, and classifications of the codes [34]. Moreover, the list allowed no code to be in 2 main groups, which facilitated the calculation of importance of each stimulus or concept referred by the codes. A selection of $30 \%$ of the scenes, equally distributed between participants and interviews, was verified with a Portuguese-born external collaborator who acts as a professional in the area of soccer. This validation reached $90 \%$ of inter-observer agreement.

The first count of code assignments was used to portray the importance of the cues, and information processing mechanisms such as judgements. The most assigned codes hinted which segments should be extracted, interpreted apart, and used as examples. The 
code assignments and extraction of examples were performed with the sole objective to identify the content of information that was taken into account during decision-making according to the narratives from the firstperson perspective. For this reason, the results were mainly limited to the content of the transcription and were only compared with the actual game images or further content from the interviews to situate actions in their context.

\section{Ethical approval}

The research related to human use has complied with all the relevant national regulations and institutional policies, has followed the tenets of the Declaration of Helsinki, and has been approved by the Ethics Committee for Research with Human Beings at Laval University.

\section{Informed consent}

Informed consent has been obtained from all individuals included in this study, as well as from their legal guardians, tutors, and the direction of the club to authorize the participation of minors and the utilization of the game images. The images and audio materials were exclusively captured for the conduction of the research in accordance with the protocol.

\section{Results}

The first game was a loss of $2-0$, and the second game was a win of 2-0. All the 3 participants played both games at their respective positions. In total, 45 actions were extracted for visualization. After the first overall analysis of the actions, it was observed that (1) most actions took place in the midfield; (2) most of the actions were directed towards the opponent's half; (3) most actions were almost exclusively passes to teammates; and (4) the actions lasted for an average of 2 seconds.

It was first revealed that the players rarely evoked tactical concepts and kept describing the events as they recalled them. This shows that the explicitation interview allowed the participants to describe without justifying their actions. It also helped the researchers identify the information that was perceived and processed during the decisional process.

The participants would mention single cues that attracted their attention. These perceptions were generally reported as direct stimuli to explain where the players would send the ball and how. However, the performers seemed to consider more than 1 element of information at the moment of decision, which re- quired specific codes. When the interviewees referred to a set of elements of information, its meaning or function was interpreted and associated with its own concept. For instance, when the participants described the movement of their team or the fact that the ball went out of the limits of the field, they referred to an idea that involved more than a single stimulus. The same interpretation was made when the interviewees qualified something they mentioned or used a reference that was not in the play. The list presented in Table 2 portrays the most recurrent and common elements of information that were identified in the narratives, for each of the groups including single or composed stimuli, information processing, and external references.

In addition to the codes presented in Table 2, the code 'space' was assigned only $6 \%$ in its code group, which reveals how rarely it is considered compared with constraints, topping 21\%. Amongst other important concepts related to performance, game model was also very scarcely referred to, notably by only 1 of the participants for 1 decision. For these reasons, both space and game model were not selected in the list of significant cues, but they will still be discussed in the next paragraphs.

\section{Interpretation}

Table 2 presents the most recurrent cues mentioned in the narratives. Thus, in extension to the first analysis, the extracts of their corresponding transcriptions were interpreted, in that case, to identify more precisely how the significant cues were taken into account in the course of action. This analysis helped understand how cues were processed and how this reflected the use of knowledge. Some extracts are provided as examples throughout the next paragraphs.

At first, it seems that the positions in the tactical system are of great use to refer to a teammate or an opponent. All the 3 players would constantly refer to the position in the system to describe what they would do, or where they would be on the field. Events of play also constitute a useful reference and, without detailing the reasons, have a direct causality on the actions of the players. Of course, the participants also mentioned available unmarked players, which in many cases would justify a pass. Nevertheless, their play was not limited to passing to one another's feet since long passes on the flanks were also observed during the games.

When considering more than 1 player to whom they could have passed, the participants would refer to the 


\section{HUMAN MOVEMENT}

G.H. Petiot, R. Bagatin, A. Mouchet, Decision-making in youth soccer

Table 2. Codes most frequently assigned to segments of the interview transcriptions

\begin{tabular}{|c|c|c|c|}
\hline Code & Definition and example & $\begin{array}{l}\text { Total } \\
\text { 'global' }\end{array}$ & $\begin{array}{l}\text { Total } \\
\text { 'during } \\
\text { action' }\end{array}$ \\
\hline \multicolumn{4}{|l|}{ Simple visual stimuli } \\
\hline Teammate's action & $\begin{array}{l}\text { Teammate's action } \\
\text { E.g.: our right back makes a pass }\end{array}$ & $18.83 \%$ & $13.61 \%$ \\
\hline Teammate's position & $\begin{array}{l}\text { Teammate's position (not in relation to the tactical system) } \\
\text { E.g.: my striker is on the edge of the box }\end{array}$ & $10.46 \%$ & $14.29 \%$ \\
\hline Configuration of play & $\begin{array}{l}\text { Description of the position of more than } 1 \text { player } \\
\text { E.g.: } 2 \text { opponents were coming towards me }\end{array}$ & $10.88 \%$ & $16.33 \%$ \\
\hline Area or depth & $\begin{array}{l}\text { Description of the position of an element of the play in relation } \\
\text { to an area of the field, e.g., depth } \\
\text { E.g.: we are going getting out of our defensive zone }\end{array}$ & $7.95 \%$ & $7.48 \%$ \\
\hline Position in the tactical system & $\begin{array}{l}\text { Position in relation to the tactical alignment } \\
\text { E.g.: our defensive mid is low }\end{array}$ & $25.94 \%$ & $28.57 \%$ \\
\hline \multicolumn{4}{|l|}{ Combined visual stimuli } \\
\hline Unmarked teammate & $\begin{array}{l}\text { Teammate who is not pressured by an opponent } \\
\text { E.g.: our striker is free }\end{array}$ & $15.32 \%$ & $23.08 \%$ \\
\hline Phase of play & $\begin{array}{l}\text { Phase or transition } \\
\text { E.g.: we were counter-attacking }\end{array}$ & $18.02 \%$ & $3.08 \%$ \\
\hline Team action & $\begin{array}{l}\text { Collective tendency: movement or tendency } \\
\text { E.g.: we are going up }\end{array}$ & $13.51 \%$ & $3.08 \%$ \\
\hline Event & $\begin{array}{l}\text { Event in the play } \\
\text { E.g.: an opponent commits a foul }\end{array}$ & $10.81 \%$ & $3.08 \%$ \\
\hline Constraint & $\begin{array}{l}\text { Limit or lack } \\
\text { E.g.: no colleague was in front of me }\end{array}$ & $12.61 \%$ & $21.54 \%$ \\
\hline \multicolumn{4}{|l|}{ Evaluation } \\
\hline Evaluation of an option & $\begin{array}{l}\text { Evaluation of an opportunity in the play } \\
\text { E.g.: the angle of pass was narrow }\end{array}$ & $68.57 \%$ & $72.73 \%$ \\
\hline \multicolumn{4}{|l|}{ External references } \\
\hline Characteristic of a colleague & $\begin{array}{l}\text { Quality of a teammate } \\
\text { E.g.: my winger is fast }\end{array}$ & - & $22.22 \%$ \\
\hline Action rule & $\begin{array}{l}\text { Rule that can be applied as a recipe } \\
\text { E.g.: when the ball comes from one side, send it the other side }\end{array}$ & - & $33.33 \%$ \\
\hline
\end{tabular}

Notes: (1) Because the interviewees always began describing the action using the ball as a reference, the ball was withdrawn from the list of stimuli. (2) Percentages are calculated in a ratio with the code assignments of the same group. The definition and a basic example are provided for each code.

closest teammates and opponents to portray a configuration of play. One, for example, said that 2 opponents were coming to pressure him. Finally, the position of the play in relation to the depth of field seemed important information. In fact, 1 participant would clearly base some decisions on how close he was from the opponent goal.

Whether passes were short or long, the participants referred to what their teammates did, especially displacements. They also described where their team- mates were in relation to the regions of the field. On some occasions, they would add details such as orientation of their teammate's body or the direction that they were pointing to. The right back was often mentioned since the player was running on the right side of the field towards the opponents' goal line. One participant indicated his teammate's individual characteristics: 'I sent the ball far into the space because I knew he would reach'. 
It was interpreted that the players did perform and report a judgement of the situation in its globality, where their own appreciation guided the decision. This corroborates the recent studies in the area, showing that the process of decision-making happens through specific mechanisms such as the evaluation of the perceived information [6]. When such judgement was shown through the explicitation of the actions, it generally reflected the following concepts.

\section{Context: time and space}

Most importantly, it was interpreted that configurations of play were translated into time constraints which would condition players to find quick solutions in order to keep ball possession. The available space being barely used as an argument reinforces the idea that constraints caused by the involvement of the opponents have more influence on the actions. Thus, reactions to these time constraints seem to portray most actions, although these actions do respect the principles of play, such as keeping ball possession and moving to offer options of pass [33]. This could be in line with the fact that many actions would consist in passing to an unmarked player, in which cases, these would be the main element of information mentioned in the interview. It could also be observed in the filmed games, for instance, that many actions were performed in interaction with the neighbouring teammates. This can be appreciated in the description of the configurations of play in the interviews as it reinforces the effect of constraints and the opportunities emerging in the play.

\section{Evaluation and risk}

In contrast, many other decisions seemed to be more calculated. The participants would also mention what the team was doing and why - mainly referring to the phase of play where the ball was starting from and where the teammates were. Such justifications can be interpreted with a higher degree of complexity as there are more cues in relation. One extract of the transcription states: 'There was an opponent on me. If I had tried to dribble him, I could have lost the ball and in that zone of the pitch, it was not very favourable'. The notion of risk - or uncertainty, as defined by the fact that the individual cannot know all probabilities and options - is manifested through such a judgement after the processing of the position of practically all the elements of the play in one single appreciation. On another occasion, a player rather evaluated: 'I didn't see anybody who I could pass to without being towards our own goal. And since we were in the third tier of the pitch, I think it was the best option'. Such a judgement let us presume that the athlete had discarded all other possibilities although they were more interesting since they were closer to the opponent goal. Finally, despite the measures taken during the interview, another player simply stated that he had sent the ball to the opposite side: 'When you receive from one side, you send it to the other one'. This also constitutes an example of conscious conceptualization that is not necessarily in line with the reality of decision-making in the play [15].

\section{Planning}

Finally, other extracts do offer a clear projection of a build-up: 'Our winger was progressing forward, and if he had passed the ball, maybe this would have ended in a dangerous play'. Referring to another situation, the player said: 'I set my right back free,' meaning that the right defender could leave his initial position to go forward. Another participant stated: 'I could have

Table 3. Map of the main observations on the participants' decisions

\begin{tabular}{llll}
\hline $\begin{array}{l}\text { Recurring concepts } \\
\text { of play }\end{array}$ & Content & Part in the game & Theoretical equivalence \\
\hline $\begin{array}{l}\text { Constraints/ } \\
\text { possibilities }\end{array}$ & $\begin{array}{l}\text { - Constraint of space and time } \\
\text { - Neighbouring teammates }\end{array}$ & $\begin{array}{l}\text { Principles of play (keep ball } \\
\text { possession, offer passing } \\
\text { options) }\end{array}$ & $\begin{array}{l}\text { Affordances [38] } \\
\text { (see comment in } \\
\text { Discussion and mplications) }\end{array}$ \\
\hline Risk & E.g.: depth of the field & $\begin{array}{l}\text { Operational principle } \\
\text { (keep ball possession) }\end{array}$ & $\begin{array}{l}\text { Representation of the play } \\
\text { [40], uncertainty [37] }\end{array}$ \\
\hline $\begin{array}{l}\text { Evaluation } \\
\text { of the option }\end{array}$ & Pertinence of the action & $\begin{array}{l}\text { Judging in accordance } \\
\text { with phase/depth }\end{array}$ & $\begin{array}{l}\text { Cognitive mechanisms (e.g., } \\
\text { anticipation, planning) [21], } \\
\text { conceptions of the game [20] }\end{array}$ \\
\hline
\end{tabular}


passed to my defenders, but I passed to my colleague whom I'd quickly seen running... Because we could have made a quick counterattack. Otherwise, we would have had to circulate the ball again'.

The examples in which the players were planning possible outcomes of their action can also be interpreted as an indicator of their representation of the play and their intentions pursued when deciding, for instance, to go in a direction or to send the ball upfront [35]. The participant stating 'I set my right back free' is using a representation in which the player he is passing to has a clear potential to bring something to the play, like helping the team to progress forward. Altogether with other notions and experimentation of constraints, these contributions give a certain sense of play to decisions. Nevertheless, this is not always the case as players do not always have the occasion to develop such a judgement in every situation since time and space will not afford it. For instance, the player explaining that he had sent the ball to the opposite side without describing details about the surrounding configuration clearly cited an easy-to-apply action rule. Such a solution would then help simplify the direction of attention and information processing. Simplified perceptions and processes are reported as a compatible solution adopted in team sports [36].

A brief version of the 4 main observations explored in the discussion is presented in Table 3.

\section{Discussion and implications}

Before making tactical knowledge a principle responsible for coherent actions, the actual opportunities of actions emerging from the play seemed to play an important role in many of the actions. Accordingly, the cognitivist framework was used as a key reference in the discussion, although the concept of affordances [38] could not be neglected. In fact, as revealed by the lived experience narratives, it was clear to the interviewees that in certain situations, there were limited if not no options to help them achieve their objective, whereas judgement and cognitive mechanisms arose when options were clearly stated.

\section{Sources of decision-making}

Certain actions could show that the players were able to process information and create relations between elements. The corresponding parts of the interviews offer a wider range of reasons justifying their actions. In these more complex situations, the participants referred to concepts that require more links between stimuli, as, for instance, the area of the field and the configuration of play. It is probable that such notions reflect the conceptions of the game, which can be used as an indicator, although knowledge is not limited to it [20]. Just as principles of play, conceptions can be of different levels of complexity since fewer players were reported to show a richer conception in a similar study about decisions [39].

Amongst these conceptions, the notion of risk and the evaluation of the situation and possible solutions would serve at the very moment of the action, and would belong to the individual as they rely on their own representation of the situation [40]. Since game modelling and specific team behaviour training are utilized to structure decision-making at a certain level of performance and at older age levels [41], such content may not apply to every decision taken by the players of this age or competitive level. In the context of this study, it can be interpreted that the adopted game model would have less impact on all decisions given the level of performance of the participants. In fact, only 1 out of the 3 players referred to directives or the game model demanded by the coach during practice. The notion of risk would then constitute an example of common references or a mental model of learning players, as a source of decision-making in a competitive environment not necessarily belonging to a team organization.

However, decisions did not seem exclusively made with respect to conceptions or notions of risk. For instance, short passing patterns for overcoming pressure and keeping ball possession would rather be caused by the passing opportunities shared between the player with the ball and the ones without the ball [42]. If the neighbouring teammates are mostly offering options of short passes, it can be assumed that the player in possession of the ball will be more likely to elect one of these short distance options when he suffers pressure. This could be explained by the limited capabilities of younger players [43] or the interpersonal coordination possibilities that the participants could actually transform into opportunities to accomplish specific objectives [44, 45]. Since coordination between movements performed in the play is communicated through motor movement itself, the complexity of decisions can increase when players depend on one another, and these decisions can change depending on who is playing because of the individual characteristics [46-48].

In the context of youth sports, players would show these characteristics in the form of capabilities like body strength or cognitive abilities. In connection with decision-making, cognition can help players apply different levels of complexity in the play. Less complex 
situations mean fewer stimuli to perceive, process, and react to. Correspondingly, fewer details were provided when actions were as simple as passing to an unmarked teammate located in proximity, whereas the participants reported experimenting dilemmas and provided more information when having to make more complex decisions [49]. It can be assumed that if the players did not specify more information on the situation, their perception of the circumstances was not as complex and therefore they did not consider the many possibilities [50]. Also, actions closer to the centre of play may be identified as less complex in their perspective than the ones requiring to comprehend the shape of the team or to bear in mind players who are far from the ball [33].

On the other hand, making situations of play and solutions simple may be one reliable way for young players to handle cognitive tasks related to decisionmaking and keep generating solutions within reach and in short timescales when under pressure. Moreover, actions performed before receiving the ball are the ones that can give players more time to act [51]. The young participants had a short practice time compared with more experienced and older players and may not have performed these actions off the ball, which resulted in less time to achieve what they wanted. In turn, other participants mentioned that they felt more comfortable in taking an action over another, which corroborates studies showing that players can prefer more effective solutions that they are confident to succeed [52]. All these considerations were additional sources of decision-making when the participants had the ball in their feet as they may not have afforded the time to perceive and process more information.

Previous studies in psychophenomenology also insist on the role of the whole body in the sports action, that is, the bodily reaction to the situation and the consequent experience. As argued earlier, it has been stressed that decisions depend on the abilities and capabilities, and hence, the body is also responsible for decisions [53]. More work on such a conception has pinpointed the importance of the embodiment of decision as a principal element of decision before cognitive processes (for a review, read [54]). Thus, decisions are to be conceptualized as a lived, embodied experience in which judgement arises depending on the situation. Further studies could employ a protocol that emphasizes the distinction between the two.

In sum, time constraints exert an important influence on decision-making and judgements in the context of play. This can make players either take incoherent actions or miss their execution, and result in limitations in their play when compared with a more complete game performance observed in advanced athletes [55]. Nevertheless, learning players' decisional background, their different skills, and the actual opportunities they can take advantage of seem to be key elements to consider when teaching the game and progressing in terms of complexity. In the context of competition, it is likely that the team will seek to keep high levels of success and require players to implement effective solutions, even if complexity increases. For instance, players who are capable of breaking down situations apply simple solutions to keep the timescale as quick as possible and can still be effective in the play. Action rules such as 'if the ball comes from one side, send it to the other side' are still part of the solutions that make players perform in the context of play as they do not hold the player back from performing.

\section{Practical implications}

The results of this study suggest 2 important elements that can direct player development in terms of tactics. First, the psychophenomenological approach to investigate the decisional background and the use of the explicitation interview had and have again shown promising outcomes concerning information that is difficult to access and describe. The implicit nature of decision-making in the context of team sports and continuous play obliges one to use appropriate techniques to describe its background and content: proceeding through the lived experience is capable of bringing enlightening results. For instance, in this study, the decisional background could be appreciated in the examples where the participants had more than one option, richer configurations of play to deal with, or even phases of play to consider. Hence, judgement calls can expectedly be richer when exploring complex situations and this gives purpose to the use of verbalization exercises [56].

In practice, it can be beneficial to re-explore decisions with the players not long after performance by making them recall their actions - for which video is largely utilized - and essentially describe them. However, there are many decisions that are not relevant to explore because they are not made with the consideration of enough constraints or possibilities emerging from the play; they are too simple. Verbalization activities are relevant and can provide hints of tactical knowledge, understanding of the game, and judgement calls where decisions are complex enough to offer a dilemma or to require a minimal set of information to put in perspective. 
Nevertheless, decisions should not be studied to be compared or discussed to validate their coherence in the play but to portray their construct. Plus, it is expected to reach limits of consciousness considering the characteristics and the experience of younger players, which can reduce the levels of details in the narratives as well as in the 'tactical thinking' as opposed to previous studies performed with professional players [9, 18]. When analysed within compatible principles and with the aim to emphasize the lived experience [57], results could still reliably inform about the decisional background and help coaches intervene more adequately. In line with the constructivist approach in sports education, such a process should help players build their understanding of the game and strive each time for better, quicker decision-making, especially through the long-term progression process in sports games [58]. In this regard, off-court activities aiming to refine decision-making are described as useful even if the nature of decisions in the play is associated with intuition [59].

The second important implication is that instructions may be very significant to players if based on their lived experience and/or concepts like the ones in Table 3. Using players' own vocabulary or referring to their reality when providing feedback or instructions would suggestively increase their efficiency. For instance, helping players better evaluate their options or manage risks may precisely appeal to their own reality without necessarily directing them to a game model. Such an approach would improve their autonomy in solving problems before converting their decision scheme into a model, which is beneficial to their development.

\section{Limitations}

This study was limited to the use of data collected with an explicitation interview as the images were only applied for the recalling of actions in the play. Such a study could be replicated with more participants, as well as with the use of images and game analysis protocols. This could help identify and emphasize a greater variability of more complex situations of play. Also, this study aimed to determine tendencies to portray elements of knowledge through a quantitative analysis. This contrasted with previous studies performed with the same method given the unicity of each narrative. Thus, further studies could employ a pure qualitative analysis protocol and only focus on the few selected situations of play, with the aim to describe their decisional background in more detail.
Besides, in respect of previous studies, the state of knowledge associated with the utilization of explicitation interview effectiveness shows that adult professional athletes were capable of describing their lived experience to the point of explaining decisions. However, limited research has brought similar results with reference to younger interviewees, such as adolescents. Therefore, it was expected that there would be differences in the narratives collected from the subjects of this study considering the dominance of prescription in the majority of training activities. Young players may have had difficulty participating in the study without prior familiarization with the interview. It would also be dangerous to come to a conclusion that the data aimed in this investigation were not obtained because the participants would not express themselves in the same way that adult sports professionals have in previous studies. We judge that, despite the limited sample and the exploratory nature of the study, the interviews have helped collect narratives of lived experience notwithstanding the tactical content of the recalled actions was expected to be limited. Moreover, the observations pinpointed in the interpretation still can be associated with the state of tactical knowledge of players of this age level. This exploratory study must be repeated to overcome the limitations with the purpose to bring more details about the situations of play and the nature of decisions. An enhanced study could aim to verify the effect of coaches' interventions [60].

\section{Conclusions}

In the description of the decisional background of learning, competitive soccer players revealed the cues that could be interpreted as significant for them at this age and level. The stimuli and concepts that were identified are in line with typical cognitive capabilities for the age level. For the aimed sample, the notion of risk in relation to the area of the pitch, the evaluation of the available options, and planning the outcome of a pass seemed to be relevant in decision-making. Nevertheless, it seems that athletes of this age, even if playing at a competitive level, still experience the strong influence of pressure. Such influence may affect their decisional process to varying degrees and prevail on judgement if they cannot afford the time to process the information. For this reason, such information can constitute a key common reference for decisional background in learning players. Nevertheless, results show that decisions also depend on the actual situations of play that the athletes are exposed to, especially in terms of opportunities and constraints. Submitting players 
to incompatible learning environment is capable of holding their development back.

Finally, this article presents the key elements of the lived experience of learning players as a possible comparable reference if using questioning approach in coaching. The psychophenomenological approach to decision-making investigation in sports or teaching the game reinforces the importance of subjectivity as it informs about the elements of the game that are significant to the performers and the part they had in decisions. This contribution can help conduct more experimental studies on decision-making to improve the player development process or work with players while applying a holistic approach [60, 61]. Finally, it adds on to the growing value of the phenomenological approach applied in sports sciences [13].

\section{Disclosure statement}

No author has any financial interest or received any financial benefit from this research.

\section{Conflict of interest}

The authors state no conflict of interest.

\section{References}

1. Vilar L, Araújo D, Davids K, Bar-Yam Y. Science of winning soccer: emergent pattern-forming dynamics in association football. J Syst Sci Complex. 2013;26(1): 73-84; doi: 10.1007/s11424-013-2286-z.

2. Matias CJ, Greco PJ. Cognition and action in team ball sports [in Portuguese]. Cienc Cogn. 2010;15(1):252271.

3. Orasanu J, Connolly T. The reinvention of decision making. In: Klein GA, Orasanu J, Calderwood R, Zsambok CE (eds.), Decision making in action: models and methods. Norwood: Ablex; 1995; 3-20.

4. De Assis JV, Costa V, Casanova F, Cardoso F, Teoldo I. Visual search strategy and anticipation in tactical behavior of young soccer players. Sci Med Footb. 2021; 5(2):158-164; doi: 10.1080/24733938.2020.1823462.

5. Casanova F, Oliveira J, Williams M, Garganta J. Expertise and perceptual-cognitive performance in soccer: a review. Rev Port Cien Desp. 2009;9(1):115-122; doi: 10.5628/rpcd.09.01.115.

6. Petiot GH, Aquino R, Cardoso F, Santos R, Teoldo I. What mental process favours quality decision-making in young soccer players? Motriz. 2017;23(3):e101735; doi: 10.1590/S1980-6574201700030003.

7. Kannekens R, Elferink-Gemser MT, Visscher C. Tactical skills of world-class youth soccer teams. J Sports Sci. 2009;27(8):807-812; doi:10.1080/026404109028 94339.

8. Práxedes A, Del Villar F, Pizarro D, Moreno A. The impact of nonlinear pedagogy on decision-making and execution in youth soccer players according to game actions. J Hum Kinet. 2018;62(1):185-198; doi: 10.1515/ hukin-2017-0169.

9. Mouchet A. Subjectivity as a resource for improving players' decision making in team sport. In: Light R, Quay J, Harvey S, Mooney A (eds.), Contemporary developments in games teaching. Abingdon: Routledge; 2013; 149-166.

10. Gesbert V, Durny A, Hauw D. How do soccer players adjust their activity in team coordination? An enactive phenomenological analysis. Front Psychol. 2017;8:854; doi: 10.3389/fpsyg.2017.00854.

11. Filho E, Tenenbaum G, Yang Y. Cohesion, team mental models, and collective efficacy: towards an integrated framework of team dynamics in sport. J Sports Sci. 2015;33(6):641-653; doi: 10.1080/02640414.2014.95 7714 .

12. Lavega-Burgués P, Luchoro-Parrilla RA, Serna J, Salas-Santandreu C, Aires-Araujo P, Rodríguez-Arregi R, et al. Enhancing multimodal learning through traditional sporting games: Marro360 ${ }^{\circ}$. Front Psychol. 2020;11:1384; doi: 10.3389/fpsyg.2020.01384.

13. Allen-Collinson J. Sporting embodiment: sports studies and the (continuing) promise of phenomenology. Qual Res Sport Exerc. 2009;1(3):279-296; doi: 10.1080/ 19398440903192340.

14. Light RL, Harvey S, Mouchet A. Improving 'at-action' decision-making in team sports through a holistic coaching approach. Sport, Education and Society. 2014;19(3): 258-275.

15. Mouchet A, Morgan K, Thomas G. Psychophenomenology and the explicitation interview for accessing subjective lived experience in sport coaching. Sport Educ Soc. 2014;19(3):258-275; doi: 10.1080/13573322.2012. 665803.

16. Raab M, Arnold A, Tielemann N. Judgment and decision making in sports: techniques for tactic training, tactics for technique training. In: Fricker P (ed.), Applied sport expertise \& learning workshop, e-version. Canberra: Australian Institute of Sport; 2005; 1-3.

17. Villemain A, Hauw D. A situated analysis of football goalkeepers' experiences in critical game situations. Percept Mot Skills. 2014;119(3):811-824; doi: 10.2466/ 25.30.PMS.119c30z0.

18. Mouchet A. Subjectivity in the articulation between strategy and tactics in team sports: an example in rugby. Ital J Sport Sci. 2005;12:24-33.

19. Práxedes A, Moreno A, Sevil J, García-González L, Del Villar F. A preliminary study of the effects of a comprehensive teaching program, based on questioning, to improve tactical actions in young footballers. Percept Mot Skills. 2016;122(3):742-756; doi: 10.1177/00315 12516649716.

20. Griffin LL, Dodds P, Placek JH, Tremino F. Middle school students' conceptions of soccer: their solutions to tactical problems. J Teach Phys Educ. 2001;20(4):324340; doi: 10.1123/jtpe.20.4.324. 
21. Ward P, Williams AM, Ericsson KA. Underlying mechanisms of perceptual-cognitive expertise in soccer. $\mathrm{J}$ Sport Exerc Psychol. 2003;25:S136.

22. Mouchet A, Vermersch P, Bouthier D. Methodology of access to subjective experience: composite interview technique and video analysis [in French]. Savoirs. 2011; 27(3):85-105; doi: 10.3917/savo.027.0085.

23. Mangas C, Garganta J, Fonseca AM. A comparative study of the declarative knowledge of young football players depending on their competitive level [in Portuguese]. In: Suarez AA, Garganta J, Lago Peñas C (eds.), Football research: the Iberian studies [in Portuguese]. Porto: Faculdade de Ciencias do Desporto e de Educação Física, Universidade do Porto; 2002; 179-187.

24. Tochon FV. Stimulated feedback, clinical objectification, shared reflection. Methodological foundations and practical applications of video feedback in research and training [in French]. Rev Sci Educ. 1996;22(3):467502; doi: 10.7202/031889ar.

25. Vermersch P. The explicitation interview. Independently published; 2007.

26. Vermersch P. Explanatory assistance and reflective feedback [in French]. Educ Perm. 2004;160:71-80.

27. Mouchet A. Characterization of the subjectivity in tactical decisions of elite rugby players [in French]. Doctoral thesis. Bordeaux: Université Victor Segalen Bordeaux 2; 2003.

28. Ericsson KA, Simon HA. Protocol analysis: verbal reports as data, revised ed. Cambridge: MIT Press; 1993.

29. Bardin L. Content analysis [in French], $2^{\text {nd }}$ ed. Paris: Presses Universitaires de France; 2016.

30. Gréhaigne J-F. Configurations of the game: debate and learning about football and team sports [in French]. Besançon: Presses Universitaires de Franche-Comté; 2007.

31. Stelter R. Experience-based, body-anchored qualitative research interviewing. Qual Health Res. 2010; 20(6):859-867; doi: 10.1177/1049732310364624.

32. Mouchet A. Articulation of scientific models to shed light on the complexity of decisions in sport [in French]. In: Quidu M (ed.), Sports science in motion: innovations and theoretical traditions in sciences and techniques of physical and sports activities [in French]. Paris: L'Harmattan; 2012; 243-268.

33. Da Costa IT, Garganta da Silva JM, Greco PJ, Mesquita I. Tactical principles of soccer: concepts and application. Motriz. 2009;15(3):657-668; doi: 10.5016/2488.

34. Anguera MT, Hernández Mendo A. Observational methodology in sport sciences. Rev Cienc Deporte. 2013;9(3): 135-160.

35. Teoldo I, Guilherme J, Garganta J. Training football for smart playing: on tactical performance of teams and players. Curitiba: Appris; 2017.

36. Raab M. Simple heuristics in sports. Int Rev Sport Exerc Psychol. 2012;5(2):104-120; doi: 10.1080/1750984X. 2012.654810.

37. Parlebas P. Games, sports, and societies: a lexicon of motor praxeology [in French]. Paris: INSEP; 1999.
38. Gibson JJ. The ecological approach to visual perception, classic edition. New York: Psychology Press; 2014.

39. González-Víllora S, García-López L-M, Gutiérrez-Díaz del Campo D, Contreras-Jordán O-R. Tactical awareness and decision making in youth football players (12 years): a descriptive study [in Spanish]. J Study Educ Dev. 2010;33(4):489-501; doi: 10.1174/021037010793 139644.

40. Oliveira JG. Specific knowledge in football. Contributions to the definition of a dynamic matrix of the teaching-learning process and game practice [in Portuguese]. Porto: Faculdade de Desporto, Universidade do Porto; 2004.

41. Schüler Giacomini D, de Oliveira Soares V, Santos HF, Matias CJ, Greco PJ. Declarative and procedural tactical knowledge in soccer players of different ages [in Portuguese]. Motricidade. 2011;7(1):43-53; doi: 10.6063/ motricidade.119.

42. Silva P, Garganta J, Araújo D, Davids K, Aguiar P. Shared knowledge or shared affordances? Insights from an ecological dynamics approach to team coordination in sports. Sports Med. 2013;43(9):765-772; doi: 10.1007/s40279-013-0070-9.

43. Borges PH, Cabral de Andrade MO, Rechenchosky L, Teoldo da Costa I, Teixeira D, Rinaldi W. Tactical performance, anthropometry and physical fitness in young soccer players: a comparison between different maturational groups. J Phys Educ. 2017;28:e2826; doi: 10.4025/jphyseduc.v28i1.2826.

44. Santos R, Duarte R, Davids K, Teoldo I. Interpersonal coordination in soccer: interpreting literature to enhance the representativeness of task design, from dyads to teams. Front Psychol. 2018;9:2550; doi: 10.3389/fpsyg.2018.02550.

45. Ribeiro J, Davids K, Araújo D, Guilherme J, Silva P, Garganta J. Exploiting bi-directional self-organizing tendencies in team sports: the role of the game model and tactical principles of play. Front Psychol. 2019;10:2213; doi: 10.3389/fpsyg.2019.02213.

46. Aguilar MP, Navarro-Adelantado V, Jonsson GK. Detection of ludic patterns in two triadic motor games and differences in decision complexity. Front Psychol. 2018; 8:2259; doi: 10.3389/fpsyg.2017.02259.

47. Gama J, Passos P, Davids K, Relvas H, Ribeiro J, Vaz V, et al. Network analysis and intra-team activity in attacking phases of professional football. Int J Perform Anal Sport. 2014;14(3):692-708; doi: 10.1080/247486 68.2014.11868752.

48. Pic M, Lavega-Burgués P, March-Llanes J. Motor behaviour through traditional games. Educ Stud. 2019; 45(6):742-755; doi: 10.1080/03055698.2018.1516630.

49. Teoldo da Costa I, Cardoso F. Football training: teaching and evaluation of tactical behaviour [in Portuguese]. In: Ramos V, Angillo Saad M, Milistetd M (eds.), Collective sports games: research and teaching practice [in Portuguese]. Florianópolis: Universidade do Estado de Santa Catarina; 2013; 107-130. 
50. Travassos B. Decision-making in futsal [in Portuguese]. Lisboa: Prime Books; 2014.

51. Oppici L, Panchuk D, Serpiello FR, Farrow D. Long-term practice with domain-specific task constraints influences perceptual skills. Front Psychol. 2017;8:1387; doi: 10.3389/fpsyg.2017.01387.

52. Musculus L, Ruggeri A, Raab M, Lobinger B. A developmental perspective on option generation and selection. Dev Psychol. 2019;55(4):745-753; doi: 10.1037/dev00 00665.

53. Davids K, Button C, Bennett S. Dynamics of skill acquisition: a constraints-led approach. Champaign: $\mathrm{Hu}-$ man Kinetics; 2008.

54. Hockey J, Collinson JA. Grasping the phenomenology of sporting bodies. Int Rev Sociol Sport. 2007;42(2): 115-131; doi: 10.1177/1012690207084747.

55. Roca A, Ford PR, McRobert AP, Williams AM. Identifying the processes underpinning anticipation and decision-making in a dynamic time-constrained task. Cogn Process. 2011;12(3):301-310; doi: 10.1007/s10339011-0392-1.

56. Raab M. Implicit and explicit learning of decision making in sports is effected by complexity of situation. Int J Sport Psychol. 2003;34(4):273-288.

57. Allen-Collinson J. Phenomenology of sensuous sporting bodies. Lincoln: University of Lincoln; 2015.

58. Jones RL, Thomas GL. Coaching as 'scaffolded' practice: further insights into sport pedagogy. Sports Coach Rev. 2015;4(2):65-79; doi: 10.1080/21640629.2016.1157321.

59. Moxley JH, Ericsson KA, Charness N, Krampe RT. The role of intuition and deliberative thinking in experts' superior tactical decision-making. Cognition. 2012; 124(1):72-78; doi: 10.1016/j.cognition.2012.03.005.

60. Jarrett K, Mouchet A, Harvey S, Scott C, Light R. Using elicitation interview within a phenomenographic framework: developing the breadth of research designs associated with game based approaches. Agora PE Sport. 2014;16(3):291-306.

61. Mouchet A. Improving decision-making in sevens rugby. In: Light R, Evans JR, Harvey S, Hassanin R (eds.), Advances in rugby coaching: an holistic approach. London: Routledge; 2015; 40-52. 